\title{
Estimation of Direction of Arrival Using Information Theory
}

\author{
Fotios Talantzis, Anthony G. Constantinides, and Lazaros C. Polymenakos
}

\begin{abstract}
Estimating the direction of arrival (DOA) of an acoustic source relies on the successful estimation of the relative delay between pairs of microphone signals. Processing is performed at the current time by operating on blocks of recorded data. When these recordings are performed in environments of strong multipath reflections, algorithms often fail to distinguish between the true DOA and that of a dominant reflection. In this letter, we assume Gaussianity of the source signal and use an information-theoretical measure, often met in blind source separation algorithms, to derive a robust DOA estimator, even under significant reverberant conditions. We discuss the most popular algorithm for time delay estimation, namely, the generalized cross-correlation method, and demonstrate under certain conditions its connection to the proposed one. Performance is demonstrated for both algorithms with sets of simulated results as a function of different reverberation times, microphone spacing, and data block size. The results indicate that the examined framework can accurately track the DOA of a typical acoustic source.
\end{abstract}

Index Terms-Audio, electroacoustics.

\section{INTRODUCTION}

A DOMINANT requirement in camera steering for automated video-conferencing is concerned with the estimation of the direction of arrival (DOA) of an acoustic source by means of time delay estimation (TDE) algorithms. Typically, the problem is approached by employing microphone arrays for the collection of data in frames so that the current TDE estimate can be provided. The most popular approach relies on defining the relative delay between a pair of microphones, which, by means of a comparing function, returns a peak at the correct DOA of the source.

The generalized cross-correlation (GCC) [1] algorithm is generally considered the most common method for TDE [2]. The delay estimate function is provided, in this case, by calculating the cross-correlation between the microphone signals and searching for the time-lag that maximizes it. The practical and, in many ways, severely restricting disadvantage of this method is that if the system is used in reverberant environments, the maximum cross-correlation could occur in a spurious delay created by the ensuing reflections. Methods to limit such effects in TDE can be found in [3] and [4]. In [5], the authors use a simple statistical model to generalize TDE performance of

Manuscript received December 23, 2004; revised February 15, 2005. The associate editor coordinating the review of this manuscript and approving it for publication was Prof. Alfred Hanssen.

F. Talantzis and L. C. Polymenakos are with Autonomic and Grid Computing Group, Athens Information Technology, GR-19002 Athens, Greece (e-mail: fota@ait.edu.gr; 1cp@ait.edu.gr).

A. G. Constantinides is with the Department of Electrical and Electronic Engineering, Imperial College London, London, SW7 2BT, U.K. (e-mail: a.constantinides@imperial.ac.uk).

Digital Object Identifier 10.1109/LSP.2005.849546
GCC variants. Other approaches used for DOA estimation include direct methods such as steered beamforming [6] and state-space approaches that employ particle filters [7].

A challenging field in audio signal processing is that of blind source separation (BSS), which uses information-theoretical measures to separate mixtures of multiple sources [8]. We employ a similar concept to provide a framework for TDE appropriate to a single acoustical source emitting in a reverberant environment. Indeed, in this framework, we use the concept of mutual information in order to create a new comparing function that calculates the correct TDE based on the maximization of information that one microphone signal has about the other. We assume that mixtures exhibit Gaussian distributions. We show that the algorithm, in order to perform correctly under various adverse reverberation conditions, must take into account the spreading of information into samples neighboring to the one that maximizes the comparing function. Thus, we propose a mathematical framework that resolves this problem and generates robust estimations under high reverberation times.

This letter is organized as follows. In Section II, we formulate the DOA estimation problem and present a variant of the GCC method, which is used at a later stage for comparison purposes. The information-theoretical alternative is presented in Section III. Section IV exhibits the performance of the two systems under different criteria, such as reverberation level, geometry, and architectural constraints of real-time systems. Section $\mathrm{V}$ summarizes the conclusions of the letter.

\section{System Model}

We concentrate on the case involving a single source and two microphones. With these in mind, consider a two-element microphone array positioned arbitrarily in an acoustical enclosure, with the microphones being $d \mathrm{~m}$ apart. The sound source is assumed to be in the far field of the array. Therefore, we can approximate the spherical wavefront emanating from the source as a plane wavefront of sound waves arriving at the microphones in a parallel manner. For the case in which the environment is nonreverberant, the assumption of a single source leads to the following discrete-time signal being recorded at the $m$ th microphone (where $m=1,2$ ):

$$
x_{m}(k)=s\left(k-\tau_{m}\right)+n_{m}(k)
$$

where $\tau_{m}$ denotes the time in samples that it takes for the source signal to reach the $m$ th microphone, and $n_{m}(k)$ is the respective additive noise (assumed to be zero mean and uncorrelated with the source signal). The overall geometry of the corresponding system can be seen in Fig. 1. Without loss of generality, this considers $m_{1}$ to be the reference microphone, i.e., $\tau_{1}=0$. The delay at $m_{2}$ is then the relative delay between the two recorded 


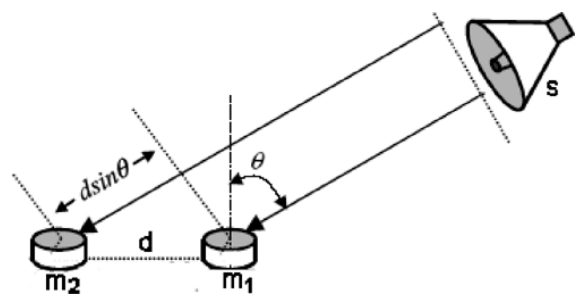

Fig. 1. Geometry of the recording system.

signals, and thus, the relationship between the microphone signals is reduced to $x_{1}(k)=x_{2}\left(k-\tau_{2}\right)$. The corresponding DOA angle $\theta$ is defined with respect to the broadside of the array and connected with any delay $\tau$ as

$$
\theta=\arcsin \left[\frac{\tau c}{f_{s} d}\right]
$$

where $f_{s}$ is the sampling frequency of the recording system, and $c$ is the speed of sound (typically defined as $343 \mathrm{~m} / \mathrm{s}$ ). Thus, the DOA can be obtained by estimating the TDE $\tau$. Note that we restrict the estimation system to integer-valued delays $\tau$, for which several of the values of $\theta$ will correspond to the same integer delay. In general, this defines the resolution of the array, and it is a function of the chosen values of $d$ and $f_{s}$.

Most of the DOA estimation techniques are required to operate in real time. We must, therefore, assume that data at each sensor $m$ are collected over $t$ frames $\widetilde{\mathbf{x}}_{m t}=\left[x_{m}(t \widetilde{L}), x_{m}(t \widetilde{L}+1), \ldots, x_{m}(t \widetilde{L}+\widetilde{L}-1)\right]$ of $\widetilde{L}$ samples. An $L$-sample subframe of $\widetilde{\mathbf{x}}_{m t}$ is denoted $\mathbf{x}_{m t}=\left[x_{m}\left(t \widetilde{L}+\nu_{1}\right), x_{m}\left(t \widetilde{L}+\nu_{1}+1\right), \ldots, x_{m}(t \widetilde{L}+\widetilde{L}-2-\right.$ $\left.\left.\nu_{2}\right), x_{m}\left(t \widetilde{L}+\widetilde{L}-1-\nu_{2}\right)\right]$, where $\widetilde{L}-\nu_{2}>\nu_{1}-1$ and $\nu_{1}, \nu_{2}=0,1, \ldots, \widetilde{L}-1$. We can, thus, shift or delay $\mathbf{x}_{m t}$ by $\nu$ samples by using the remaining $\nu_{1}+\nu_{2}$ samples. We denote this as $\mathbf{x}_{m t}(\nu)$. Thus, $\widetilde{\mathbf{x}}_{m t}=\mathbf{x}_{m t}$ for $L=\widetilde{L}$ (i.e., $\nu_{1}=\nu_{2}=0$ ) and $\mathbf{x}_{m t}(0)=\mathbf{x}_{m t}$. Since the analysis will be independent of the data frame, we can drop $t$ to express frames simply as $\mathbf{x}_{m}$ for any $t$. We use subframes in order to allow a representation of the data received at the microphone array as a function of a delay $\tau$. In the context of our model, and for any set of frames, we may then write $\mathbf{x}_{1}=\mathbf{x}_{2}\left(\tau_{2}\right)$. Thus, the problem is to estimate the correct value of $\tau=\tau_{2}$ and the DOA by processing two subframes $\mathbf{x}_{1}$ and $\mathbf{x}_{2}(\tau)$ only.

The DOA is typically obtained by using the original GCC function, as in [1], or one of its variants. The GCC-PHAT version $R(\tau)$ is defined as the cross-correlation of $\mathbf{x}_{1}$ and $\mathbf{x}_{2}(\tau)$, filtered by a weighting function $\mathrm{g}$ for a range of delays $\tau$ that determine the size of $L$ so that delayed or shifted versions of the subframes do not require samples from previous or future frames. If $\mathbf{X}_{1}(\omega), \mathbf{X}_{2}(\omega ; \tau)$ and $\mathbf{G}(\omega)$ denote the corresponding $L$-point discrete Fourier transforms, we can express $R(\tau)$ as

$$
R(\tau)=\frac{1}{\pi} \sum_{\omega} \mathbf{G}(\omega) \mathbf{X}_{1}(\omega) \mathbf{X}_{2}^{*}(\omega ; \tau) e^{j \omega \tau}
$$

with

$$
G(\omega)=\frac{1}{\left|\mathbf{X}_{1}(\omega) \mathbf{X}_{2}^{*}(\omega ; \tau)\right|}
$$

Ideally, $R(\tau)$ exhibits a global maximum at the lag value that corresponds to the correct $\tau$. Thus, an estimation of $\tau_{2}$ can be obtained by $\tau_{2}=\arg \max _{\tau} R(\tau)$. This can then be converted to the corresponding angle $\theta$ according to (2). GCC-PHAT is considered to be the most robust version of GCC [2], and it is, thus, chosen here for performance comparisons.

However, in a real environment, the model of (1) fails to include the effect of reverberation. We can model this as

$$
x_{m}(k)=h_{m}(k) * s(k)+n_{m}(k)
$$

with $h_{m}(k)$ representing the reverberant impulse response between the source and the $m$ th microphone. The $*$ symbol denotes convolution. $h_{m}(k)$ is now really a filter that contains not only the effect of $\tau_{m}$ (direct path) but also that of several other delays created by the reverberant paths. The length of $h_{m}(k)$-and, thus, the number of reflections - is a function of the reverberation time $T_{60}$ (defined as the time in seconds for the reverberation level to decay to $60 \mathrm{~dB}$ below the initial level) of the room.

The GCC-PHAT algorithm is able to return accurate estimates of the relative delay when the environment is described by the anechoic model of (1). However, it has a major drawback when used in an environment described by (5). The true source location will correspond to a peak in $R(\tau)$. Nevertheless, in the presence of reflections, this peak may not always be the global maximum. This is particularly the case when $T_{60}$ is not relatively low. It is, thus, of interest to devise a method that remains robust under such conditions.

\section{INFORMATION-THEORETICAL DELAY ESTIMATION}

Bell et al. [8] presented a way of separating sound mixtures of multiple sources in a blind manner, by minimizing the mutual information (MI) between them. We use the same concept but tailor it appropriately to the tracking of an acoustic source.

In general, the MI of two variables is an information-theoretical measure that represents the difference between the measured joint entropy of the two signals and their joint entropy if they were independent. Without loss of generality, we may consider $\mathbf{x}_{1}$ and $\mathbf{x}_{2}(\tau)$ to be stationary stochastic processes, for which the MI between them is defined as [9]

$$
I=H\left[\mathbf{x}_{1}\right]+H\left[\mathbf{x}_{2}(\tau)\right]-H\left[\mathbf{x}_{1}, \mathbf{x}_{2}(\tau)\right]
$$

where $H\left[\mathbf{x}_{m}\right]$ is the differential entropy of $\mathbf{x}_{m}$, and $H\left[\mathbf{x}_{1}, \mathbf{x}_{2}(\tau)\right]$ is the joint entropy of $\mathbf{x}_{1}$ and $\mathbf{x}_{2}(\tau)$.

The problem of finding the correct relative delay between the two signals is equivalent to finding the delay $\tau$ that maximizes (6). Intuitively, this means that when we determine this delay and synchronize the two microphone signals, the information that one microphone signal has about the other will be maximum. If we assume that the source signal is zero-mean Gaussian distributed, the MI is equal to the following [9]:

$$
I=-\frac{1}{2} \ln \frac{\operatorname{det}[\mathbf{C}(\tau)]}{C_{11} C_{22}}
$$


with $\operatorname{det}[\cdot]$ as the determinant operator and $\mathbf{C}(\tau)$ as the joint covariance matrix of the microphone signals. For sufficiently large $L$ (ideally $L \rightarrow \infty) \mathbf{C}(\tau)$ can be approximated as

$$
\mathbf{C}(\tau) \approx\left[\begin{array}{c}
\mathbf{x}_{1} \\
\mathbf{x}_{2}(\tau)
\end{array}\right]\left[\begin{array}{c}
\mathbf{x}_{1} \\
\mathbf{x}_{2}(\tau)
\end{array}\right]^{T}=\left[\begin{array}{cc}
C_{11} & C_{12}(\tau) \\
C_{21}(\tau) & C_{22}
\end{array}\right]
$$

Note that $C_{11}$ and $C_{22}$ are time-shift independent variables. The relative delay is obtained as that delay that maximizes (7), i.e., $\tau_{2}=\arg \max _{\tau}\{I\}$.

There is a theoretical equivalence between maximizing the MI in (7) and the GCC algorithm. Indeed, maximizing (7) is equivalent to minimizing $\operatorname{det}[\mathbf{C}(\tau)]$ or simply equivalent to finding the delay $\tau$ that maximizes the modulus of the off-diagonal terms of $\mathbf{C}(\tau)$. This is, in fact, the time-domain interpretation of the basic form of the GCC method.

Consequently, the MI-based estimator suffers from the same limitations of GCC and its PHAT variant, i.e., it would not be robust enough in multipath environments. One would wonder, though, why an information-based measure is subject to the same problem. This may be explained as follows. In the case of the anechoic model, only a single delay is present in the microphone signals. Thus, the measurement of information contained in a sample $l$ of $\mathbf{x}_{1}$ is only dependent on the information contained in sample $l-\tau$ of $\mathbf{x}_{2}(\tau)$. In the case of the reverberant model, though, information contained in a sample $l$ of $\mathbf{x}_{1}$ is also contained in neighboring samples of sample $l-\tau$ of $\mathbf{x}_{2}(\tau)$ due to the convolution operator. Thus, the MI calculation of (7) is not representative enough in the presence of reverberation. The same logical argument applies to the samples of $\mathbf{x}_{2}(\tau)$. Thus, in order to estimate the information between the microphone signals, we use the marginal MI that considers jointly $N$ neighboring samples and can be formulated as follows [9]:

$$
\begin{gathered}
I_{N}=H\left[\mathbf{x}_{1}\right]+H\left[\mathbf{x}_{1}(1)\right]+\ldots+H\left[\mathbf{x}_{1}(N)\right]+H\left[\mathbf{x}_{2}(\tau)\right] \\
+H\left[\mathbf{x}_{2}(\tau+1)\right]+\ldots+H\left[\mathbf{x}_{2}(\tau+N)\right] \\
-H\left[\mathbf{x}_{1}, \mathbf{x}_{1}(1), \ldots, \mathbf{x}_{1}(N), \mathbf{x}_{2}(\tau), \mathbf{x}_{2}(\tau+1)\right. \\
\left.\ldots, \mathbf{x}_{2}(\tau+N)\right]
\end{gathered}
$$

which, after lengthy but straightforward manipulations, reduces to the following expression for the Gaussian case:

$$
I_{N}=-\frac{1}{2} \ln \frac{\operatorname{det}[\mathbf{C}(\tau)]}{\operatorname{det}\left[\mathbf{C}_{11}\right] \operatorname{det}\left[\mathbf{C}_{22}\right]}
$$

with the joint covariance matrix

$$
\begin{aligned}
\mathbf{C}(\tau) & \approx\left[\begin{array}{c}
\mathbf{x}_{1} \\
\mathbf{x}_{1}(1) \\
\vdots \\
\mathbf{x}_{1}(N) \\
\mathbf{x}_{2}(\tau) \\
\mathbf{x}_{2}(\tau+1) \\
\vdots \\
\mathbf{x}_{2}(\tau+N)
\end{array}\right]\left[\begin{array}{c}
\mathbf{x}_{1} \\
\mathbf{x}_{1}(1) \\
\vdots \\
\mathbf{x}_{1}(N) \\
\mathbf{x}_{2}(\tau) \\
\mathbf{x}_{2}(\tau+1) \\
\vdots \\
\mathbf{x}_{2}(\tau+N)
\end{array}\right]^{T} \\
= & {\left[\begin{array}{cc}
\mathbf{C}_{11} & \mathbf{C}_{12}(\tau) \\
\mathbf{C}_{21}(\tau) & \mathbf{C}_{22}
\end{array}\right] . }
\end{aligned}
$$

If $N$ is chosen to be greater than zero, the elements of $\mathbf{C}(\tau)$ are now themselves matrices. In fact, for any value of $\tau$, the size of $\mathbf{C}(\tau)$ is always $2(N+1) \times 2(N+1)$. For the purposes of the present letter, we call $N$ the order of the tracking system. Note that for $N=0$, the marginal MI function reduces to the original relationship (7). We show experimentally in the following section that increasing the order improves DOA estimation. The range of values of $\tau$ and the chosen $N$ determine the size of $L$ so that the delayed or shifted versions of the subframes do not require samples from previous or future frames.

When (10) reaches a maximum as a function of $\tau$ at a specific time shift, then there is at this point a joint process with a maximum transport of information between $\mathbf{x}_{1}$ and $\mathbf{x}_{2}(\tau)$. According to the presented information-theoretical criterion, this is the delay that synchronizes the two recordings. In the context of DOA, this delay returns the correct angle $\theta$, at which the signal coincides with the microphone array.

\section{SYSTEM DisCUSSION}

We conducted DOA estimation simulations for a single source to a two-microphone system. A male speech signal of duration $5 \mathrm{~s}$ sampled at $f_{s}=44.1 \mathrm{kHz}$ is used. The relative sample delay that the systems is to estimate varies according to $d$. Since the system can only cope with integer delays, the optimal scenario for the system is to estimate these delays as the rounded nearest integer.

Experiments were performed for three different environments distinguished by their reverberation times $T_{60}$. For the used sampling rate $f_{s}$, these result in impulse responses $h(k)$ of different lengths. The impulse responses are generated using the image model [10] modified to allow for noninteger sample delays. The simulated room dimensions are $[5,4,3] \mathrm{m}$. These were then convolved with the speech signal to create the microphone signals. Moreover, $15 \mathrm{~dB}$ of additive noise was also introduced to the signals. The process was repeated for ten random displacements and rotations of the relative geometry between the source and the receivers inside the room. The rest of the environment variables are given below.

\begin{tabular}{|c|c|}
\hline Variable & Value \\
\hline Distance between source & \\
and midpoint of receivers $(m)$ & 2.23 \\
Actual delay (samples) & 6.89 \\
Expected delay (samples) & 7 \\
$T_{60}(\mathrm{~s})$ & $0.15,0.30,0.50$ \\
Length of $h(k)$ (samples) & $6615,13230,22050$ \\
\hline
\end{tabular}

For each frame of data processed, the system returns an estimate $\widehat{\tau}$ from which the current DOA is obtained. The squared error for frame $t$ is then computed as

$$
\sigma_{t}=\left(\tau-\widehat{\tau}_{t}\right)^{2} .
$$

The root mean-squared error (RMSE) metric is the performance measure used to evaluate the system. For a single displacement of the geometry, this is defined to be the square root of the average value of $\sigma_{t}$ over all frames. In the figures to follow, 


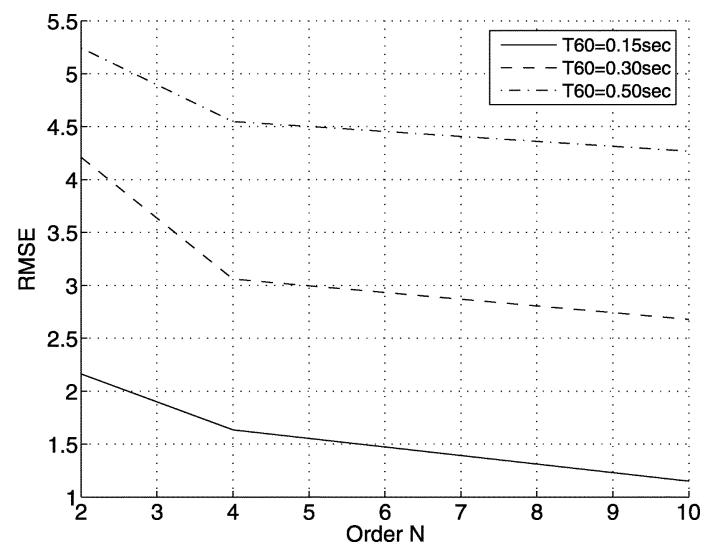

Fig. 2. RMSE versus order $N$ for different values of $T_{60} . L=0.5 \times T_{60} f_{s}$.

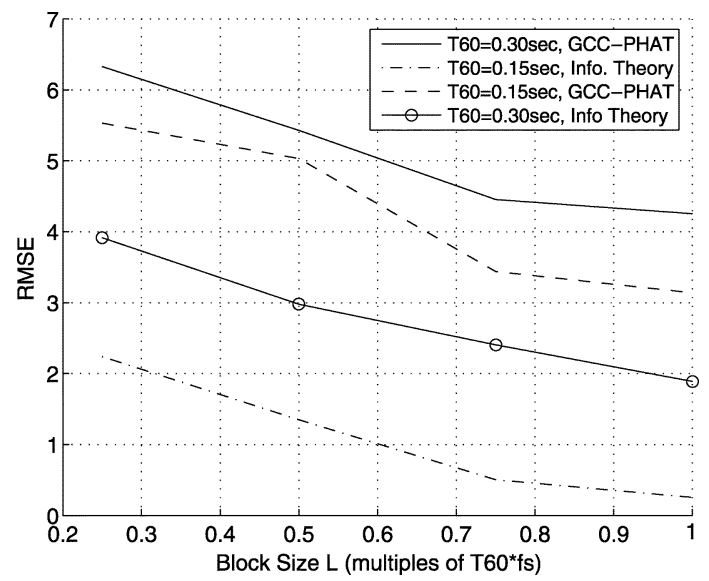

Fig. 3. RMSE of MI and GCC-PHAT systems for varying $L$ (shown for $T_{60}=$ $0.15 \mathrm{~s}$ and $T_{60}=0.30 \mathrm{~s}$ ). The microphone spacing is $0.12 \mathrm{~m}$.

we present the average RMSE over all ten simulations. Thus, the lower the average RMSE value, the better the performance of the estimating system.

Choosing the order $N$ of the delay estimation system affects the performance of the system. Fig. 2 shows the RMSE for varying $N$ in all three environments. $L$ is chosen to be $0.5 \times$ $T_{60} f s$. Increasing the order $N$ decreases the RMSE since the $\mathrm{MI}$ and the resulting delay estimations become more accurate.

DOA estimation systems are designed to operate in real time, and a crucial characteristic is the size of $L$ to be selected. In real systems, an accurate estimate of $\theta$ should be given repeatedly after small segments of time. At the same time, algorithms require enough data to be able to include the effect of reverberation in their estimations. Thus, we examine the effect of the size of $L$ by considering a series of different block sizes. These are $L=[0.25,0.5,0.75,1] \times T_{60} f_{s}$ in samples. Fig. 3 expresses the effect of $L$ on the performance of the presented method as compared to GCC-PHAT for $T_{60}=0.15 \mathrm{~s}$ and $T_{60}=0.30 \mathrm{~s}$. This shows that, for the chosen $T_{60}$ and $L$, the information theory-based method is more robust than its GCC counterpart. In all cases, $N=10$ and frames were windowed using a Hamming window. In real-time systems, where small block sizes are required, the presented system would obviously be preferable since it requires far fewer data to perform satisfactorily.

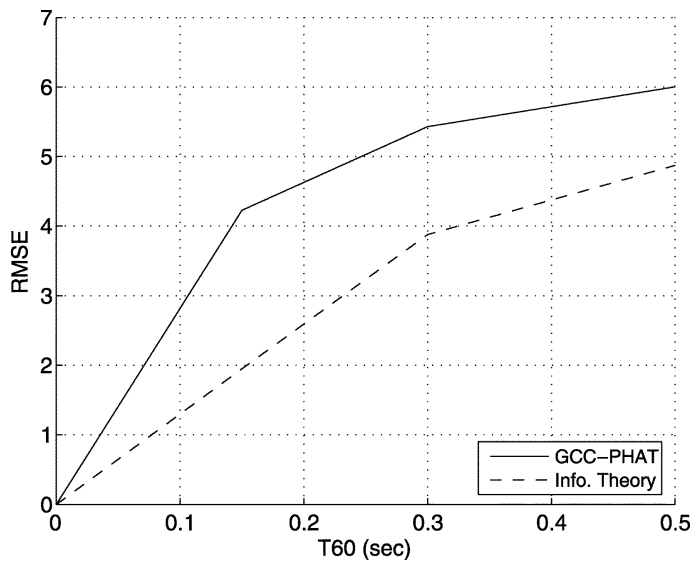

Fig. 4. RMSE of MI and GCC-PHAT systems for varying T60. $L=0.5 \times$ $T_{60} f_{s}$ (shown for microphone spacing of $0.12 \mathrm{~m}$ ).

Another performance factor is the effect of reverberation. As the room becomes more reverberant, the performance of the estimating systems gets degraded because reflections get mistaken for the actual DOA of the source. Fig. 4 summarizes the effect for the case when $L=0.5 \times T_{60} f_{s}, N=10$. Again, the presented method outperforms the GCC-PHAT approach.

\section{CONCLUSION}

In this letter, we presented a DOA estimator based on the use of MI concepts. We demonstrated that under certain conditions, it is equivalent to the simple GCC method and presented a way of embedding information about reverberation so that estimations are more robust and consistent. This was verified by simulated results, for which the modified MI framework showed adequate robustness for the examined relative geometry and any combination of block size and $T_{60}$.

\section{REFERENCES}

[1] C. H. Knapp and G. C. Carter, "The generalized correlation method for estimation of time delay," IEEE Trans. Acoust., Speech, Signal Process., vol. ASSP-24, no. 4, pp. 320-327, Aug. 1976.

[2] M. Brandstein and D. B. Ward, Microphone Arrays Signal Processing Techniques and Applications. New York: Springer-Verlag, 2001.

[3] P. Aarabi and S. Mavandadi, "Multi-source time delays of arrival estimation using conditional time-frequency histograms," Inf. Fusion, vol. 4, no. 2, pp. 111-122, 2003.

[4] M. S. Brandstein and H. Silverman, "A robust method for speech signal time-delay estimation in reverberant rooms," in Proc. IEEE Int. Conf. Acoust., Speech, Signal Process., 1997, pp. 375-378.

[5] T. Gustafsson, B. D. Rao, and M. M. Trivedi, "Source localization in reverberant environments: modeling and statistical analysis," IEEE Trans. Speech Audio Process., vol. 11, no. 6, pp. 791-803, Nov. 2003.

[6] B. D. Van Veen and K. M. Buckley, "Beamforming: a versatile approach to spatial filtering," IEEE ASSP Mag., vol. 5, no. 2, pp. 4-24, Apr. 1988.

[7] D. B. Ward, E. A. Lehman, and R. C. Williamson, "Particle filtering algorithms for tracking an acoustic source in a reverberant environment," IEEE Trans. Acoust., Speech, Signal Process., vol. 11, no. 6, pp. 826-836, Nov. 2003.

[8] A. Bell and T. Sejnowski, "An information maximization approach to blind separation and blind deconvolution," Neural Comput., vol. 7, pp. 1129-1159, 1995.

[9] T. M. Cover and J. A. Thomas, Elements of Information Theory. New York: Wiley, 1991.

[10] J. B. Allen and D. A. Berkley, "Image method for efficiently simulating small-room acoustics," J. Acoust. Soc. Amer., vol. 65, no. 4, pp. 943-950, 1979. 